\title{
Stationen einer Passion
}

\author{
Sandra Ziegler
}

PhD, MS, Geschäftsführerin EMH seit Juni 2018

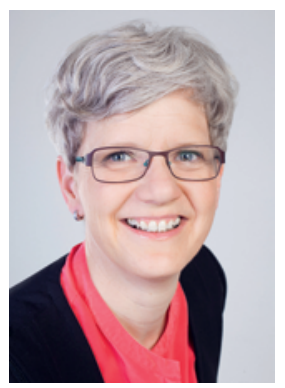

Im Frühsommer 1998 stand ich in der Innenstadt von St. Gallen und wusste, dass ich eine Passion gefunden hatte. Es war Abstimmungskampf zur Gen-SchutzInitiative, und ich als Zellbiologie-Doktorandin engagierte mich an Standaktionen, um den Passantinnen und Passanten die Konzepte, Werkzeuge und Chancen der Gentechnologie verständlich zu machen. Die Initiative wurde abgelehnt, wir gehörten zu den Gewinnern. Für mich wog jedoch eine Erkenntnis auf persönlicher Ebene fast stärker: Ich hatte viel Spass dabei, diese komplexen wissenschaftlichen Inhalte runterzubrechen, in einen neuen, alltäglichen Kontext zu stellen, anders zugänglich zu machen. Dabei wollte ich bleiben.

Ich hatte viel Spass, diese komplexen wissenschaftlichen Inhalte runterzubrechen und in einen neuen, alltäglichen Kontext zu stellen.

In der Folge führte ich das kleine Science Museum psi forum am Paul Scherrer Institut und vermittelte anhand von Exponaten und Führungen die PSI-Themen - von der Energieforschung bis zu neuen Brennstoffzellen, vom Teilchenbeschleuniger bis zur Protonentherapie für Augentumore. Ich glaube, dass wir am PSI mit diesen Aktivitäten sehr viele Leute ansprechen und auch begeistern konnten, in der Schweiz aber zu den Pionieren zählten. Ausserhalb der Schweiz, vor allem im angelsächsischen Raum, waren die Aktivitäten in der Wissenschaftskommunikation schon viel etablierter, war der Erfahrungsschatz breiter. Ich entschloss mich daher - ausgestattet mit einem Stipendium des Schweizerischen Nationalfonds -, mich an der Boston University in Wissenschaftsjournalismus und Public Relations weiterzubilden. Nach dem Studiengang und ausgerüstet mit wertvollen Erkenntnissen und vielen neuen Impulsen, leitete ich von Boston aus die globale interne Kommunikation der Forschungseinheit NIBR von Novartis. Zurück in der Schweiz, wechselte ich wieder in ein akademisches
Forschungsumfeld. Ich kommunizierte für das Wasserforschungsinstitut EAWAG neue Erkenntnisse für den Gewässerschutz oder Lösungen für sanitäre Probleme in Entwicklungsländern und baute in den letzten Jahren am Friedrich Miescher Institut in Basel die Kommunikationsaktivitäten auf und aus - von Web, über Social Media, bis zur eher klassischen Medienarbeit.

Bei all diesen Aktivitäten stand im Zentrum, wie wir die zum Teil äusserst anspruchsvollen Themen attraktiv für die verschiedenen Zielgruppen - Forschende und Laien, Mitarbeitende in verschiedenen Ländern, Politikerinnen und Politiker, Medienschaffende - aufbereiten. Was für Bedürfnisse haben die verschiedenen Personen, was für einen Hintergrund bringen sie mit, was ist relevant für sie? Dazu mache ich mir nach wie vor mit viel Enthusiasmus Gedanken, entwickle Strategien und setze diese dann gerne abwechslungsreich um.

Als neue Geschäftsleiterin der EMH bringe ich diese Passion und meine Erfahrung in diesem Bereich ins Team. Ich sehe unsere Aufgabe auch in diesem Kontext: Wir stellen als redaktionell unabhängiger Verlag attraktive und relevante Inhalte in verschiedenen

\section{Ich freue mich auf die Zusammenarbeit inner-} halb der EMH und mit den verschiedenen

Vertretern aus dem Gesundheitswesen.

Formaten den Ärztinnen und Ärzten in der Schweiz zur Verfügung. Wir stellen mit den Medizinerinnen und Medizinern in den Redaktionen sicher, dass wir erstklassige Fortbildung bieten, und wir stärken mit qualitativ hochstehenden Beiträgen den Dialog zwischen den verschiedenen Akteuren und unter den Peers. Ich freue mich auf die Zusammenarbeit innerhalb der EMH und mit den verschiedenen Vertretern aus dem Gesundheitswesen und bin gespannt auf die Herausforderungen, die sich aus dem kompetitiven Umfeld ergeben, in welchem sich EMH bewegt. 